\title{
Clinical and Histopathological Evaluation of MDP/Collagen Induced Arthritis Rat Model (MCIA) after Treatment with Urtica Dioica, Plantago Major and Hypericum Perforatum L Herbal Mixture
}

\author{
Mohammad S. KHALIFEH ${ }^{1,3)}$, Wael HANANEH ${ }^{2)}$, Raida AL-RUKIBAT ${ }^{2)}$, \\ Omar OKOUR ${ }^{1)}$, and Assia BOUMEZRAG ${ }^{1)}$
}

\begin{abstract}
1)Department of Basic Veterinary Medical Science, Veterinary Science College, Jordan University of Science and Technology, Irbid, ${ }^{2}$ Department of Veterinary Pathology and Animal Health, Veterinary Science Collage, Jordan University of Science and Technology, Irbid, and ${ }^{3}$ Department of Molecular Biology and Genetic Engineering, Science College, Jordan University of Science and Technology, Irbid, Jordan
\end{abstract}

\begin{abstract}
This study was done to assess the effects of Urtica dioica, Plantago major and Hypericum perforatum $L$ herbal mixture in the MCIA rat model. In addition, a new pathological and clinical arthritis lesion assessment was developed. Sprague-Dawley (SD) rats were immunized with bovine type I/ collagen and muramyl dipeptide (MDP). Commercial herbal extracts were administered daily to the rats after the immunization for the course of experiment (90 days). Rats were boosted with a second collagen-MDP emulsion 60 days after the first immunization. Paws were daily evaluated macroscopically for redness, swelling, distortion, or ankylosis of the joints. On the day of sacrifice, rat paws were assessed for histopathologic changes. Herbal mixture administration decreased the clinical lesion manifestation in the MCIA rat model and led to development of similar or slightly more severe histopathological lesions compared to rats that did not receive the treatment. The clinical arthritis signs appeared as early as 13 days after the first MDP/collagen injection and with peak incidence at 20 days post-immunization. Histopathologically, animals showed changes ranging from mild to very severe. Administration of the herbal mixture used in this study had a clinical therapeutic effect on the course of the clinical manifestations in the MCIA model, but the herbal treatment had no such effect on the histopathological lesion development and even led to slightly more severe lesions. Rats in the MCIA model developed prominent clinical and histopathological changes that were comparable to rheumatoid arthritis (RA) lesions in humans.
\end{abstract}

Key words: collagen-induced arthritis, Hypericum perforatum L, muramyl dipeptid, Plantago major, Urtica dioic

(Received 20 February 2007 / Accepted 25 October 2007)

Address corresponding: M.S. Khalifeh, Department of Basic Veterinary Medical Science, Veterinary Science College, Jordan University of Science and Technology, P. O. Box 3030 Irbid, 22110 Jordan 


\section{Introduction}

Collagen induced arthritis (CIA) is an experimental model which resembles human RA in its pathological and immunological features [7]. This model is widely used to study the etiopathogenesis of RA aiming to evaluate effective therapies for this disease [7,11]. Like RA, CIA is characterized by rapid onset of joint inflammation followed by cartilage destruction and bone erosion. Early histopathological changes in RA are prominent infiltration of mononuclear cells in the synovial sublining layer of the joint [8]. In more advanced stages of RA, joint capsule thickens [16] followed by pannus tissue formation that invades the joint tissue component, leading to destruction of articular bone and cartilage [13]. Later, fibrous tissues obliterate the joint cavity space resulting in more sophisticated complications of RA such as finger deformity as well as fibrous ankylosis of the joint [16]. The clinical course of RA fluctuate extremely with episodes of remission and worsening [4, 8]. Locally, the disease leads to symmetrical swelling and tenderness of peripheral joints on palpation, motion impairment and morning stiffness [4, 8]. Moreover, fatigue, weight loss, pain, malaise and fever are most often clinical signs associated with RA [4, 8]. The clinical presentations of RA vary according to the affected joints and the stage of the disease [4]. Early rheumatoid arthritis is characterized by symmetric polyarthritis, a feature which often affects the small joints of hands and feet [4]. Progressive lesion development results in joint instability and deformities [4].

Folklore medicines have gained great popularity in the past few years. Several plants have been tested for their effectiveness in the treatment of many diseases. Medicinal herbs such as Urtica dioica, Plantago major and Hypericum perforatum $L$ were part of some of these preparations. The crude herbal extracts of these herbs or specific components of the extracts have been shown to have anti-inflammatory effects mostly in vitro. Leaf extracts from Urtica dioica acts by switching Th1 derived responses to Th2; therefore, it may inhibit inflammatory events of RA [14]. In addition, it has been reported in a pilot study of 40 patients with RA that the combination of $50 \mathrm{mg}$ of Urtica dioica with $50 \mathrm{mg}$ of diclofenac showed similar effectiveness to $200 \mathrm{mg}$ di- clofenac and this is important for patients suffering from non steroidal anti-inflammatory drugs (NSAIDs) side effects [3]. Moreover, oral treatment of mice and rats with aqueous extract of dried Plantago major leaves inhibited prostaglandin synthesis [15]. Plantago major leave extract also inhibited the development of paw oedema in rats injected with carrageenan through inhibition of cyclooxygenase activity [17]. Hypericum perforatum $L$ as well as other hypericum species are known for their analgesic and antidepressant activities [2,10]. This medicinal herb has also been used to relieve pain and inflammation [12].

In this study, we investigated the effect of Urtica dioica, Hypericum perforatum $L$ and plantago major herbal extract mixture on the clinical and histopathological development of arthritis induced by injection of collagen type II and (MDP) in a SD rat model. Here, we show that this herbal mixture led to delayed onset and decrease in clinical signs severity, but that it was not successful in decreasing the disease incidence and the local histopathological changes associated with arthritis. Instead, at the herbal mixture dose and treatment duration used in this study, the incidence and histopathological changes were exacerbated in the herbal treated group compared to the group that had only arthritis without herbal treatment.

\section{Materials and Methods}

\section{Collagen preparation and arthritis induction}

Arthritis was induced according to the protocol recommended by Chondrex Inc. (Redmond, WA). Briefly, lyophilized bovine type II collagen extracted from the nasal septum cartilage (Sigma, St. Louis, MO) was dissolved in $0.05 \mathrm{M}$ acetic acid at a concentration of 4 $\mathrm{mg} / \mathrm{ml}$ by gentle stirring overnight at $4^{\circ} \mathrm{C}$. The collagen was then combined at a ratio $1: 1$ with $2.5 \mathrm{mg} / \mathrm{ml} \mathrm{N}$ acetylmuramyl-alanyl-D-isoglutamine hydrate or so called muramyl dipeptide (MDP) adjuvant (Sigma, St . Louis, MO). The collagen-MDP solution was further emulsified with an equal volume of incomplete Freund's adjuvant (IFA) (Sigma, St. Louis, MO) in a drop wise fashion with continuous stirring with electric homogenizer. The stability of the emulsion was tested by adding one drop of emulsion into a beaker of water. A stable 
emulsion remained, as a solid clump in water without dispersing, while the spreading onto the water surface indicated an unstable emulsion. The final collagen/MDP/ IFA emulsion was kept cold on ice and administered by injection to animals within two hours of preparation.

Arthritis was induced by a subcutaneous injection of $0.2 \mathrm{ml}$ of the cold emulsion with a Hamilton glass syringe into the base of the tail, $2-3 \mathrm{~cm}$ from the body on day 0 . Rats were boosted subcutaneously by a second injection of $0.1 \mathrm{ml}$ of the collagen emulsion also into the base of the tail 60 days after the first immunization.

\section{Herbal extraction and herbal treatment}

Standardized commercial herbal extracts of flowering herb top of Hypericum perforatum (St. John's Wort), leaf extracts of Urtica dioica (Nettle) and leaf extracts of Plantago major (Plantain) were purchased from a global herbal supplier (Mareeba, Australia). The alcohol content of each extract was evaporated and the evaporated quantities were reconstituted with distilled water. Herbal extracts in this prescription were mixed at 1:1:1 ratio. Rats were daily treated with the maximum tolerized dose $(1 \mathrm{ml})$ orally using an intragastric gavage for the whole period of experiment (90 days).

\section{Animal subjects and experimental design}

Sprague-Dawley (SD) male rats, at an age between six to ten weeks were assigned to 4 experimental groups. The SD strain was originally purchased from B\&K Universal Limited (Grimston, Aldbrough, England). Rats for this work were obtained from breeding colonies maintained under controlled conditions at the Biological Center of Research (Jordan University of Science and Technology). The animals were maintained under a climate controlled environment at $24^{\circ} \mathrm{C}$ and an alternating $12 \mathrm{~h}$ light-dark cycle (8:00 to 20:00). Each rat was housed individually in a metallic cage containing wood shavings, fed standard rodent chow and allowed tap water ad libitum. Treatment and maintenance were in accordance with the guidelines of the Jordan University of Science and Technology Animal Care and Use Committee (JUST-ACUC) which follows the international animal care and use guidelines (ILAR). Animals in MCIA $\& \mathrm{Hb}$ (herbal) group (16 animals) received a daily dose of herbal extract mixture and were subcutaneously in- jected with collagen/MDP emulsion as previously described; MCIA group (16 animals) were injected only with collagen/MDP emulsion; Hb group ( 8 animals) only received a daily dose of herbal extract mixture and control group (10 animals) only received distilled water daily and served as a negative control group.

\section{Evaluation of clinical arthritis}

Animals were observed daily for signs of arthritis and hind paw thickness was measured by caliper twice weekly. The severity of inflammation in each paw was evaluated everyday according to the degree and extent of erythema and edema of the periarticular tissue, as well as the swelling, distortion, or joint ankylosis. The clinical severity was assessed by calculation of the arthritis index according to a scoring system described by Ingrid Marty (2001) as follows: 1 point for each swollen digit except the thumb, 1 point for the carpal or tarsal joint, 1 point for the metacarpal or metatarsal joint with a maximum score of 6 for a hind paw and 5 for a forepaw. The arthritis index was obtained by summing the scores recorded for each limb. Therefore, the maximum cumulative clinical arthritis scores per rat was 22 points [9]. A modification of this scoring system was introduced which considered division of clinical signs into mild and severe changes. Arthritis clinical lesions were considered severe if the scoring index was above 11 points meaning involvement of 2 affected paws with a diameter increase in paw thickness that reached at least $0.3 \mathrm{~mm}$ above normal. The presence of joint deformities, which is usually not accompanied by prominent swelling that cannot be translated as an increase in paw diameter, was also categorized in the severe group. Incidence percentage was calculated through dividing the number of animals with any clinical signs of arthritis over the total of the number animals in each group at specific time points. The clinical severity percentage was calculated through dividing the number of animals with severe signs of arthritis over the number of arthritic animals in each experimental group. Two animals from the MCIA group died on days 32 and 64, and one animal from MCIA \& Hb group died on day 64 .

\section{Histopathological examination}

The affected interphalangeal (IP) joints were removed 
and longitudinally dissected following euthanasia using carbon dioxide inhalation, chamber method. Tissue samples were fixed in $10 \%$ buffered formalin for $24 \mathrm{~h}$ and decalcified using 5\% formic acid for two weeks, washed with tap water, processed and embedded in paraffin blocks. Tissue sections were cut at $5 \mu \mathrm{m}$ thickness and stained with hematoxylin and eosin ( $\mathrm{H} \& \mathrm{E})$, then examined histopathologically.

\section{Histopathological scoring system}

Distal IP joints were always the first joints to become inflamed, followed by the proximal IP joints. The sections of digits were prepared to ensure that proximal, middle, and distal phalanges with their opposing cortices and cartilage ends were present. Histological sections of inflamed hind paw interphalangeal joints were scored on the basis of the presence of each of the following criteria: fibroblast hyperplasia, synoviocytes hyperplasia, osteoblast hyperplasia, capsule thickness with inflammatory cells infiltration, new blood vessel formation, prominent osteoclast, osteoid seams, pannus formation, new bone formation, bone destruction and joint ankylosis. At least, ten H\&E slides sections were scored at $4 \times$, $10 \times$ and $40 \times$ for these parameters. Each one of the previous parameters was scored as follows: no detectable change (grade 0); slight change (grade 1); moderate change (grade 2) and severe change (grade 3). The arthritic disease was categorized into 3 main stages, early stage, advanced stage I and advanced stage II as follows: lesions with only fibroblast hyperplasia, synoviocytes hyperplasia and osteoblast hyperplasia regardless of grade were considered as the early stage; lesions with capsule thickness along with inflammatory cells infiltration, angiogenesis, prominent osteoclast and osteoid seams at any grade as well as pannus formation, new bone formation, bone destruction or joint ankylosis present with grade 1 or 2 was considered as the advanced stage I; and lesions with either pannus formation, new bone formation, bone destruction or joint ankylosis present with grade 3 was categorized as the advanced stage II.

\section{Results}

\section{The incidence and severity of arthritis}

Rats developed gradual onset of arthritis from day 13 post collagen emulsion administration with a peak incidence on day 30. Most animals showed severe arthritic lesions within one week to ten days; however, some animals developed severe lesions very rapidly within $24 \mathrm{~h}$ of arthritis onset. The maximum percentage of rats that showed arthritic lesions in the group receiving MDPcollagen emulsion was $66 \%$ (MCIA group); while that of the group that received herbal extracts mixture along with emulsion was 50\% (MCIA \& $\mathrm{Hb}$ ) 30 days post collagen emulsion injection (Fig.1A). On day 60 post arthritis induction, the percentage of animals that developed arthritic lesions in both these experimental groups became equal (53\%) (Fig. 1A). At this point, another dose of the emulsion was given to all animals in these two groups. Unexpectedly, the booster injection caused a dramatic increase in the incidence of arthritis in the MCIA \& Hb group. The percentage of animals that had arthritic lesions in this group became $73 \%$ whereas the MCIA group had only a slight increase in the incidence percentage after the second injection (57\%) (Fig. 1B).

The first signs of arthritis were always observed in the hind limbs. Typically, the affected limb became severely red and swollen within $24 \mathrm{~h}$ in the ankle, tarsal, metatarsal and interphalangeal joints (Fig. 2A). This was represented by an increase in the dorso-plantar as well as the latero-median diameter of the metatarsophalangeal joint region, at least two times above normal diameter. The swelling persisted for 4 to 5 weeks then disappeared completely in some mildly affected animals. However, the swelling in the majority of severely affected animals was transformed to clear deformities of the paws. (Fig. 2B).

The arthritic lesion severity observed macroscopically was more pronounced in animals from the MCIA group than in the animals that showed arthritic lesions in the MCIA \& Hb group. Therefore, the percentage of animals that showed severe arthritic lesions in animals with any signs of arthritis in the MCIA and MCIA \& $\mathrm{Hb}$ groups were $100 \%$ and $60 \%$, respectively. These changes were apparent as early as one week following the first sign of lesion manifestation (Fig. 3A). It was also noted 
(A)

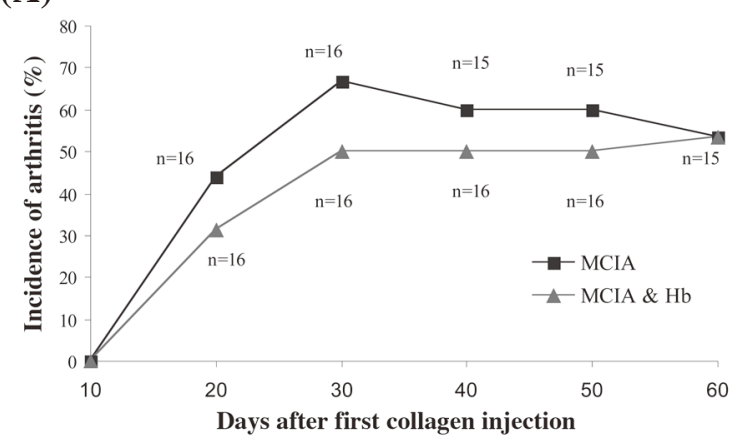

(B)

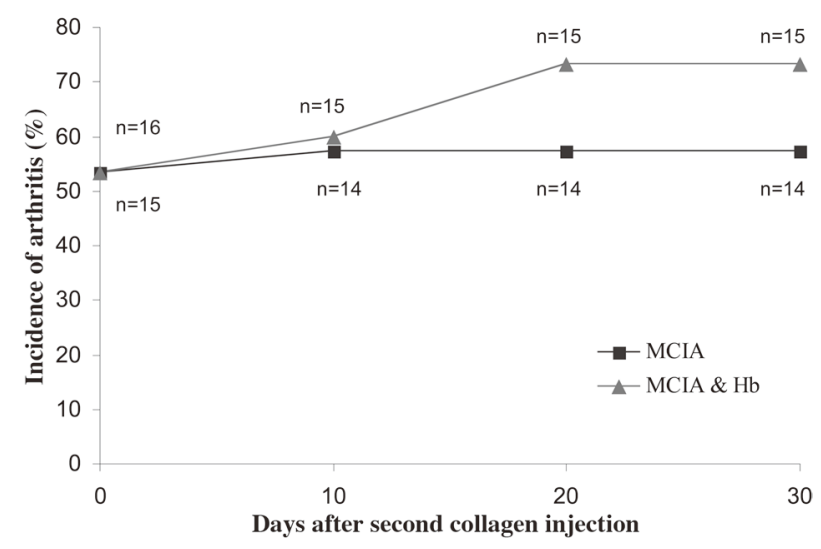

Fig. 1. Percentage of rats that developed arthritis from day 10 after the first subcutaneous injection with $200 \mu \mathrm{l}$ of collagen-MDP-IFA emulsion at the base of the tail (A). Percentage of rats that developed arthritis after a second subcutaneous injection with $100 \mu \mathrm{l}$ of collagen-MDP-IFA emulsion (B). Rats in the MCIA \& Hb group intragastrically received a daily dose of $1 \mathrm{ml}$ herbal mixture.

that the severity decreased with time in both experimental groups. A marked decrease in severity was observed in the MCIA \& Hb group (37\%) on the day of the second MDP/collagen/IFA emulsion injection (day 60) compared with $71 \%$ in the MCIA group (Fig. 3A). After the $\mathrm{MDP} /$ collagen/IFA booster emulsion injection, the severity was dramatically increased in the animals that did not receive herbal mixture and reached $83 \%$ one week after the second MDP/collagen/IFA emulsion injection. In contrast, the percentage of animals with severe lesions in the MCIA \& Hb group was constant around $36 \%$ and did not change until the end of the experiment (30 days post second injection) (Fig. 3B).
(A)

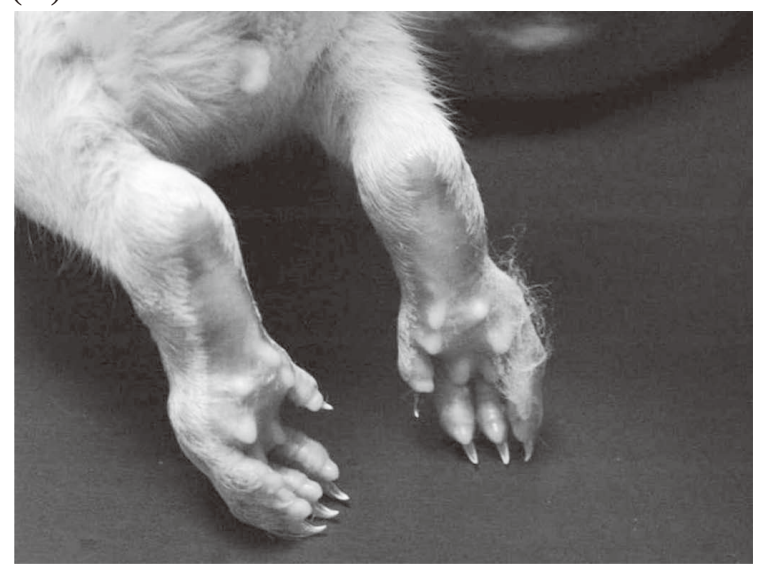

(B)

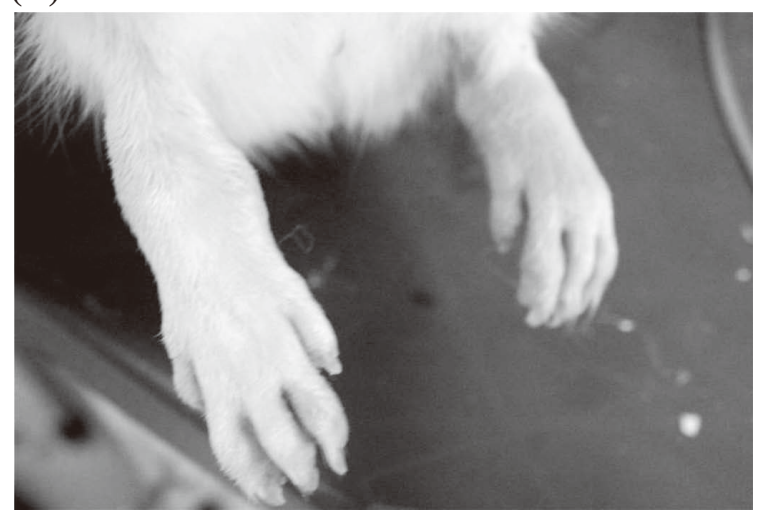

Fig. 2. Photograph taken 5 days after arthritis onset (A). Arthritis affected both hind limbs of a rat immunized with collagen/MDP/IFA. Severe swelling and redness in the tarsal, metatarsal and interphalangeal joints. Photograph taken 90 days post arthritis induction (B). Clear deformities in the hind limb from an arthritic rat immunized with collagen/MDP/IFA.

\section{Histopathological findings}

Histopathological changes were categorized into 2 main stages: early stage and advanced stage. Also the advanced stage was subgrouped into advanced stage I and advanced stage II. Ninety days after the first MDP/ collagen/IFA emulsion injection, interphalangeal joints were assessed histopathologically. The unaffected cases showed no significant histopathological findings and no indication of either cartilage or bone lesions were seen. The articular surfaces were smooth with regular contour. The joint capsules were within normal limits. The early stage of the disease showed variable degrees of synovial cell hyperplasia and hypertrophy. The mildest changes consisted of a few layers of thickening of 

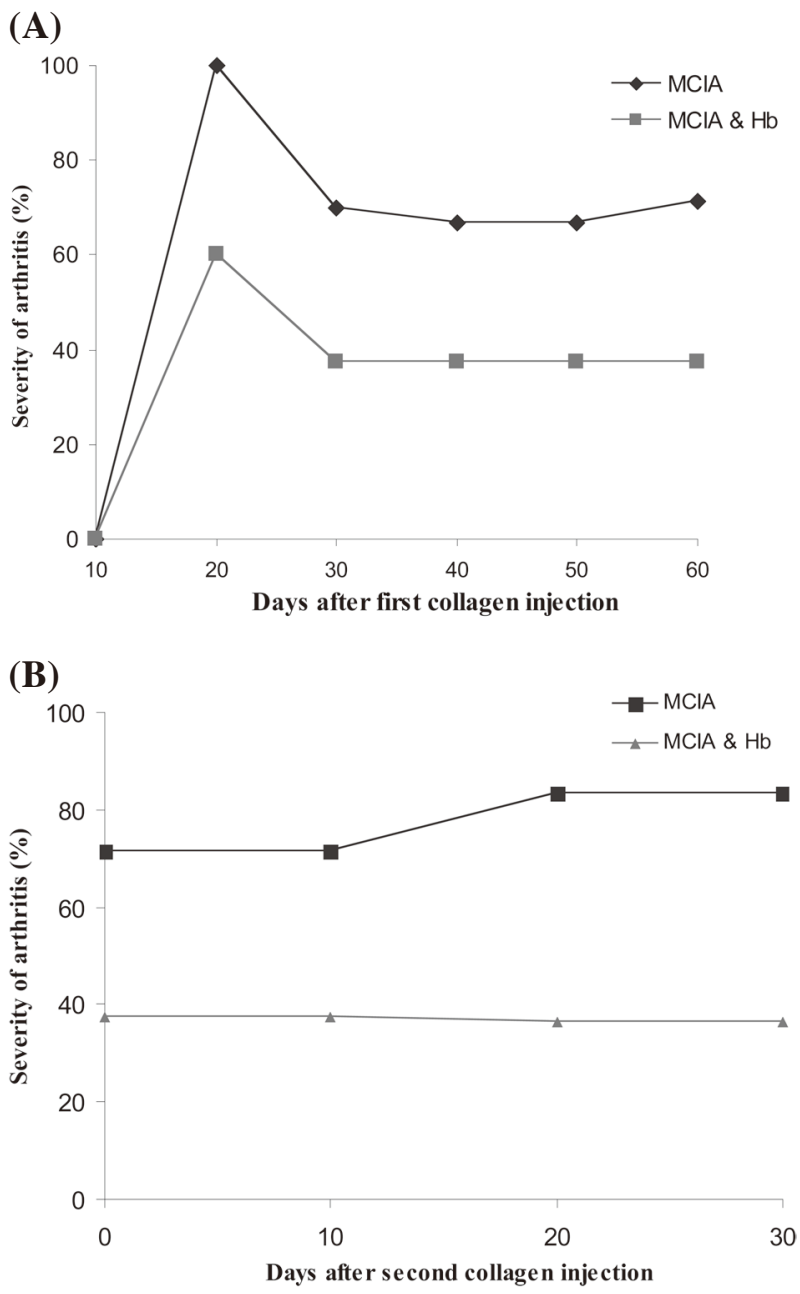

Fig. 3. Percentage of animals with severe clinical arthritic lesion among the animals that developed arthritis starting 10 days after the first collagen-MDP-IFA emulsion injection (A). Percentage of animals with severe clinical arthritic lesion among the animals that developed arthritis within 30 days after the second collagen-MDP-IFA emulsion injection (B). Rats in the MCIA \& Hb group intragastrically received a daily dose of $1 \mathrm{ml}$ herbal mixture.

the synoviocyte, up to 3-4 layers. In the most severe cases, 10 or more layers of hypertrophied synoviocytes were seen. Also in this stage, hyperplasia and hypertrophy of osteoblasts were frequently present and the most severely affected cases had very prominent osteoblasts. Advanced stage I was characterized by different degrees of capsular thickenings with fibroplasia and pleocellular infiltrates (Fig. 4). Mononuclear cells predominated. In severe cases, the capsule was tremendously thickened up to $8-10 \times$ thicker than normal counterparts with or

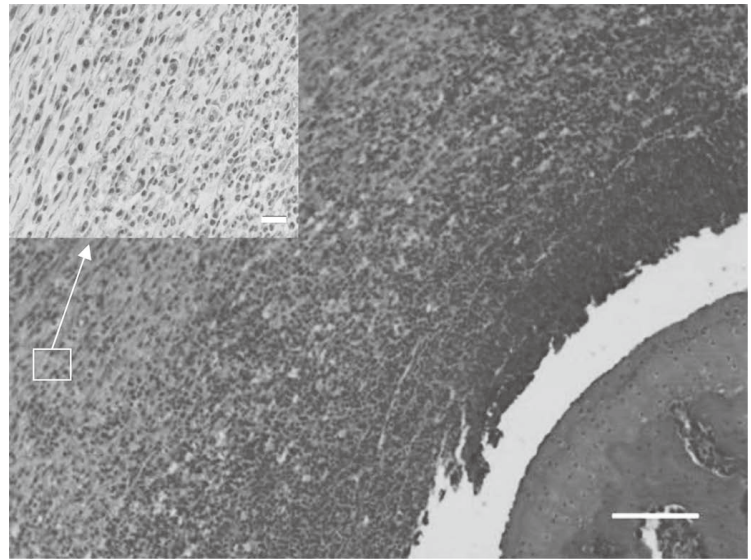

Fig. 4. Interphalyngeal joint, Sprague-Dawley rat. The joint capsule is 10 times thicker than normal due to fibroblast proliferations and large numbers of mixed inflammatory cell infiltrates. HE. Bar=10 $\mu \mathrm{m}$. Inset shows the inflammatory cell components: neutrophils, macrophages, plasma cells and a few lymphocytes admixed with fibroblasts. HE. Bar $=20 \mu \mathrm{m}$.

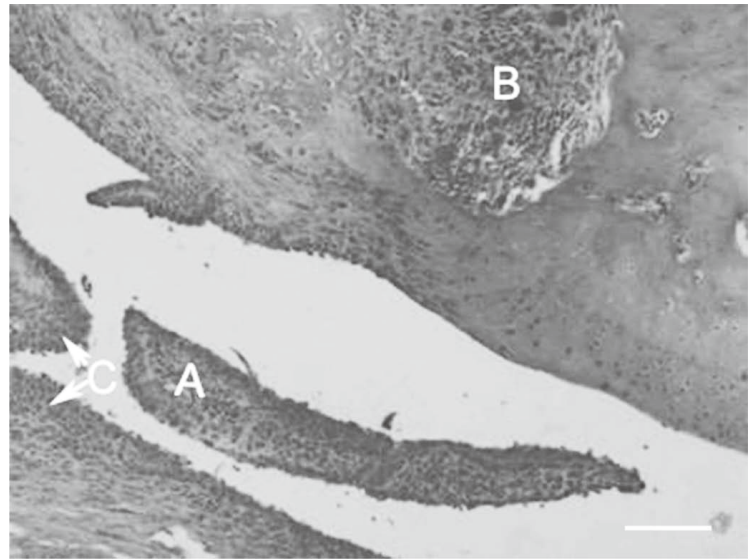

Fig. 5. Interphalyngeal joint, Sprague-Dawley rat. Pannus formation within the joint space (A). Articular surface erosion and necrosis by pannus and prominent osteoclasts (B). Note the thickening of the synoviocyte layers (C) HE. Bar=10 $\mu \mathrm{m}$.

without inflammatory cell infiltrates. Furthermore, in this stage, Osteoclasts and osteoid seams were prominent, indicative of bone remodeling. Osteoclasts were seen with marked indentations within the bone. In advanced stage II, pannus formation, articular cartilage erosion and bone destruction characterized this stage (Fig. 5). In severe cases, complete obliteration of the normal architecture of the joint space with pannus formation that in 


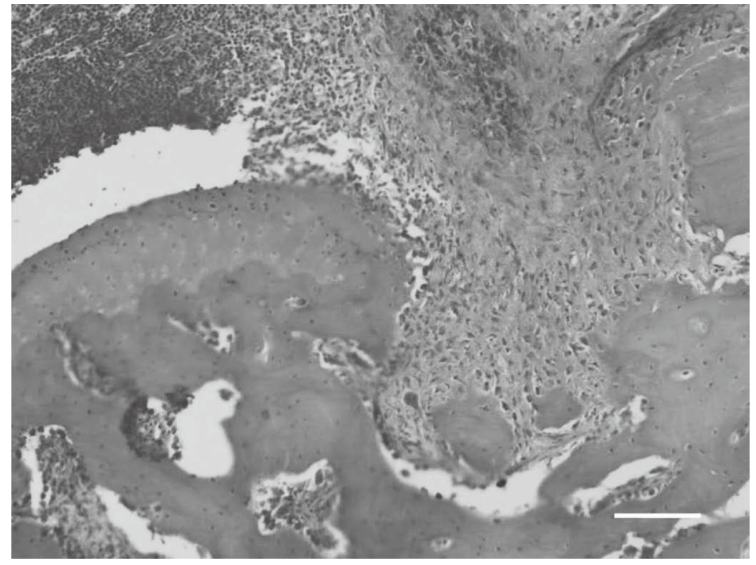

Fig. 6. Interphalyngeal joint, Sprague-Dawley rat. The joint space is completely obliterated with pannus formation that erodes and invades the subchondral bone. HE. Bar= $10 \mu \mathrm{m}$.

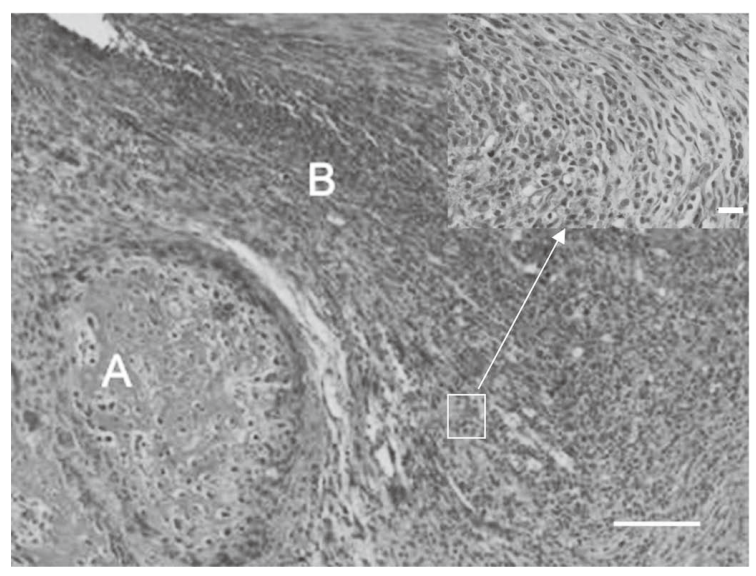

Fig. 7. Interphalyngeal joint, Sprague-Dawley rat. New bone formation (A). Severe capsule thickening with severe inflammatory cell infiltrates (B). HE. Bar $=10 \mu \mathrm{m}$. Inset shows large number of neutrophils, macrophages, plasma cells and a few lymphocytes infiltrating the thickened capsule. HE. Bar=20 $\mu \mathrm{m}$.

many cases eroded the articular cartilages and invaded the subchondral bone resulted in joint ankylosis as complete or partial (Fig. 6). In some cases, variable sized new bone formation was seen in different affected areas in the bone primarily within the pannus (Fig. 7).

\section{Histopathological arthritis incidence and lesion severity percentages}

Thirty days after the booster MDP/collagen/IFA emul-
Table 1. Incidence percentages of histopathological lesions in two experimental groups

\begin{tabular}{lcc}
\hline Animal group & MCIA \& Hb & MCIA \\
\hline Total number & 15 & 13 \\
Arthritic rats & 15 & 11 \\
Incidence percentage & $100 \%$ & $85 \%$ \\
\hline
\end{tabular}

Table 2. Lesion severity percentage in the MCIA group (a total of 11 rats showing histopathological arthritic lesions)

\begin{tabular}{lccc}
\hline \multirow{2}{*}{ Stage } & \multirow{2}{*}{ Early stage } & \multicolumn{2}{c}{ Advanced stage } \\
\cline { 3 - 4 } & & I & II \\
\hline Number of arthritic animals & 3 & 3 & 5 \\
Percentage severity & $27.3 \%$ & $27.3 \%$ & $45.4 \%$ \\
Percentage severity (Total) & $27.3 \%$ & $72.7 \%$ & \\
\hline
\end{tabular}

Table 3. Lesion severity percentage in the MCIA\&Hb group (a total of 15 rats showing histopathological arthritic lesions)

\begin{tabular}{lccc}
\hline \multirow{2}{*}{ Stage } & \multirow{2}{*}{ Early stage } & \multicolumn{2}{c}{ Advanced stage } \\
\cline { 3 - 4 } & & I & II \\
\hline Number of arthritic animals & 3 & 5 & 7 \\
Percentage severity & $20 \%$ & $33.3 \%$ & $46.7 \%$ \\
Percentage severity (Total) & $20 \%$ & $80 \%$ & \\
\hline
\end{tabular}

sion injection (day 90), the incidence and severity of arthritis was calculated according to the histopathological changes. All animals in the control group and in the group that received herbal mixture alone showed no significant histopathological changes in the interphalangeal joints. As a result, the arthritis score parameters for these two groups were grade 0 . All the animals $(n=15)$ in the group that received herbs along with the MDP/collagen/IFA emulsion injections had histopathological arthritic lesions while in the MCIA group ( $n=14)$ only $85 \%$ of the animals showed arthritic lesions (Table 1). In the MCIA group, the percentage of rats that showed arthritic lesions in the early stage of arthritis was $27.3 \%$, while the percentage of rats that showed arthritic lesions in advanced stages I and II was $27.3 \%$ and $45.4 \%$, respectively (Table 2). In the MCIA and $\mathrm{Hb}$ treated group, the histopathological lesion severity was $20 \%$ in the early stage, $33.3 \%$ for advanced stage I and $46.7 \%$ for advanced stage II (Table 3). 


\section{Discussion}

Collagen induced arthritis is considered a good model for studying the pathogenesis of RA. It has been successfully induced in many sensitive strains of mice such as DBA/1 and B10-RIII and rats such as BB and Wistar Furth [5]. However some other animal strains develop arthritis with great variability in incidence, onset and severity. In the present study, immunization of an inbred strain of SD rats, which is considered a less sensitive strain, with type II collagen-MDP induced severe arthritis starting as early as 13 days post injection. A very severe form of arthritis developed which reached its peak 20 days post injection (100\%) with affection of the four limbs in $86 \%$ of animals (data not shown). Therefore, the combination of (MDP) with collagen type II resulted in increasing the severity of arthritis in SD rats, a strain which is less sensitive to CIA. The present study focused on a rat CIA model because of the many shared features between this animal model and human RA [7]. In order to determine the different histopathological lesions of the interphalangeal joint, different sections of the joints were evaluated and scored with a new scoring system. This system took into consideration the developmental stages of the pathological lesions, with lesions classified into three stages: early stage, advanced stage I and advanced stage II.

According to this system, animals that had only fibroblast hyperplasia, synoviocytes hyperplasia and osteoblast hyperplasia regardless of grade (1 to 3 ) were classified pathologically as the early stage of the disease. This classification is based on the pathological changes that are usually reported in early RA [16]. These changes include synovial cell proliferation with congestion of the synovial membrane as well as infiltration of polymorphonucleated cells and lymphocytes [16]. The presence of capsule thickness with inflammatory cells infiltration, angiogenesis, prominent osteoclast and osteoid seams at any scoring grade as well as pannus formation, new bone formation, bone destruction or joint ankylosis present with a grade of 1 or 2 were classified as advanced stage I. This is in agreement with what has been reported for the advanced stages of RA, in which the histopathological lesions are characterized by marked fibroplasia with thickening of joint capsule [16]. Moreover, in chronic inflamed synovial tissue, the joint space is obliterated with pannus as well as fibrous tissue formation [16]. Animals with pannus formation, new bone formation, bone destruction or joint ankylosis present of grade 3 were classified as advanced stage II. This is consistent with many investigations which have observed that in the very late stages of RA, the pannus extends and invades the subchondral bone, leading to small marginal bone erosion [1,9]. With disease progression, further spreading of the pannus into bone is present and results in more bone destruction [13]. This is also in agreement with the changes that have been reported as a common complication in late stage RA in which deviation and deformity of fingers, as well as fibrous ankylosis of the joint are prominent features [16].

Folklore medicine has gained great popularity worldwide. Therefore, many researchers have directed their studies to the most commonly used plants in the treatments of some chronic diseases. However, most of these studies were carried in vitro on commercial standardized extracts and lacked properly designed scientific clinical trials which usually address any adverse effects due to the misuse of these herbs. In the present study, three herbal extracts were combined and administered daily to rats that have the potential to develop arthritis due to the injection of MDP/collagen/IFA emulsion. The herbal extracts were used at a dose that was tested to be nontoxic to these animals (data not shown). Rats that received the herbal mixture developed arthritis more readily and their number increased especially after the second emulsion injection but with low clinical severity.

In addition, administration of a second dose of MDP/ collagen/IFA caused a recrudescence of the disease within 10 days in two previously mild arthritic rats which became severe. None of the animals which remained non-arthritic after first injection developed the clinical disease when re-challenged. Therefore, the second injection did not have any effect on the incidence. However, after the second injection a slight increase in the clinical severity $(71-83 \%)$ in the MCIA group was observed. This finding corroborates the results of previous findings which reported that Lewis rats receiving a booster injection of type II collagen 6 months after the first immunization showed a recurrence of symptoms in all previously affected animals within 3 days [6]. 
The histopathological findings in the two MCIA groups, MCIA group and MCIA \& Hb group, revealed that the incidence percentage of rats that showed arthritic histopathological lesions was $100 \%$ in the MCIA $\& \mathrm{Hb}$ group and $85 \%$ in the MCIA group. Although this incidence was higher than what was reported clinically in this experiment, this incidence percentage still has a trend similar to that reported clinically. In addition, it is normal to find new cases in histopathology that were not apparent clinically.

In the current study, the histopathological findings revealed that the percentages of rats that showing arthritic lesions and classified in the early stage of arthritis among the total number of animals with some pathological changes were $27.3 \%$ in the MCIA group and 20\% in the MCIA \& Hb group. Furthermore, the percentages of rats showing severe arthritic histopathological lesions and classified in the advanced stages was similar in both these experimental groups, with a slightly higher percentage in the MCIA \& Hb group (80\%) than in the MCIA group (72.7\%). This histopathological finding disagreed with the clinical findings. This is not of great impact, since the employed clinical scoring system in this study accounted for changes that were present in all the joints and not on a separate joint lesion. In other words, we can conclude that the clinical severity of the disease decreased, in terms of number of joints involved, with the herbal treatment but when evaluating lesions histopathologically, the severity was similar in the two MCIA treatment groups regardless of the herbal treatment.

In conclusion, it is of great importance that more accurate and comprehensive evaluation of the biological effects of medicinal plants on health and disease in vivo is undertaken before recommending such herbs for medication. Regarding this point, the herbal combination used in this study might carry a risk of increasing the incidence of arthritis in people at high risk. However, it seems that in well developed arthritic cases, the use of such herbs might decrease the distribution of the clinical signs and therefore reduce the clinical disease severity. It is important to further purify the active gradients of each herb and then investigate the effect as well as the use of each herb alone and not in a mixture with different therapeutic doses in future work.

\section{Acknowledgment(s)}

This work was supported by a grant from Deanship of Research, Jordan University of Science and Technology. Authors acknowledge the technical help of Mr. Mohammad Damreh at the Biological Center of Research (animal house).

\section{References}

1. Ainola, M.M., Mandelin, J.A., Liljestrom, M.P., Li, T.F., Hukkanen, M.V., and Konttinen, Y.T. 2005. Pannus invasion and cartilage degradation in rheumatoid arthritis: involvement of MMP-3 and interleukin-1beta. Clin. Exp.Rheumatol.23: 644-650.

2. Apaydin, S., Zeybek, U., Ince, I., Elgin, G., Karamenderes, C., Ozturk, B., and Tuglular, I. 1999. Hypericum triquetrifolium Turra. extract exhibits antinociceptive activity in the mouse. J. Ethnopharmacol. 67: 307-312.

3. Chrubasik, S., Enderlein, W., Bauer, R., and Grabner, W. 1997. Evidence for antirheumatic effectiveness of Herba Urticae dioicae in acute arthritis: a piolte study. Phytomedicine 4: 105-108.

4. Grassi, W., De Angelis, R., Lamanna, G., and Cervini, C. 1998. The clinical features of rheumatoid arthritis. Eur. J. Radiol. 27: S18-S24.

5. Griffiths, M.M. 1988. Immunogenetics of collagen induced arthritis in rats. Int. Rev. Immunol. 4: 1-15.

6. Guerne, P.A., Zuraw, B.L., and Vaughan, J.H.J. 1989. Synovium as a source of interleukin 6 in vitro. Contribution to local and systemic manifestations of arthritis. J. Clin. Invest. 83: 585-592.

7. Joe, B. and Wilder, R. 1999. Animal models of rheumatoid arthritis. Mol. Med. Today 5: 367-369.

8. Lee, D.M. and Weinblatt, M.E. 2001. Rheumatoid arthritis. Lancet 358: 903-911.

9. Marty, I., Peclat, V., Kirdaite, G., Salvi, R., So, A., and Busso, N. 2001. Amelioration of collagen-induced arthritis by thrombin inhibition. J. Clin. Invest. 107: 631-640.

10. Muller, W.E., Rolli, M., Schafer, C., and Hafner, U. 1997. Effects of hypericum extract (LI 160) in biochemical models of antidepressant activity. Pharmacopsychiatry 30: 102-107.

11. Myers, L.K., Rosloneic, E.F., Cremer, M.A., and Kang, A.H. 1997. Collagen-induced arthritis, an animal model of autoimmunity. Life Sci. 61: 1861-1878.

12. Ozturk, B., Apaydin, S., Ince, E.G.I., and Zeybek, U. 2002. Hypericum triquetrifolium Turra. Extract exhibits antiinflammatory activity in the rat. J. Ethnopharmacol. 80: 207-209.

13. Resnick, D. and Petterson, H. 1992. Skeletal radiology. In: NICER Series on Diagnostic Imaging, Merit Comm., London.

14. Riehemann, K., Behnke, B., and Schulze-Osthoff, K. 1999. Plant extracts from stinging nettle (Urtica dioica), an 
antirheumatic remedy, inhibit the proinflammatory transcription factor NF-kappaB. FEBS Lett. 442: 89-94.

15. Samuelsen, A.B. 2000. The traditional uses, chemical constituents and biological activities of Plantago major L. A review. J. Ethnopharmacol. 71: 1-21.
16. Scutellari, P.N. and Orzincolo, C. 1998. Rheumatoid arthritis: sequences. Eur. J. Radiol. 27: S31-S38.

17. Shipochliev, T., Dimitrov, A., and Aleksandrova, E. 1981. Anti-inflammatory action of a group of plant extracts. Vet. Med. Nauki 18: 87-94. 